Revue d'histoire de l'Amérique française

REVUE D.HISTOIRE DE L'AMÉRIQUE FRANÇAISE

\title{
La découverte de Montréal par Jacques Cartier
}

\section{Claude Perrault}

Volume 20, numéro 2, septembre 1966

URI : https://id.erudit.org/iderudit/302568ar

DOI : https://doi.org/10.7202/302568ar

Aller au sommaire du numéro

Éditeur(s)

Institut d'histoire de l'Amérique française

ISSN

0035-2357 (imprimé)

1492-1383 (numérique)

Découvrir la revue

Citer cet article

Perrault, C. (1966). La découverte de Montréal par Jacques Cartier. Revue d'histoire de l'Amérique française, 20(2), 236-261.

https://doi.org/10.7202/302568ar d'utilisation que vous pouvez consulter en ligne.

https://apropos.erudit.org/fr/usagers/politique-dutilisation/ 


\section{LA DÉCOUVERTE DE MONTREAAL PAR JACQUES CARTIER *}

Quand nous consultons les documents du 16ème, du 17ème, et même du 18ème siècle, pour certains pays, nous constatons que les dates des événements varient à cause du calendrier employé par ces différents royaumes. ${ }^{1}$ Comme Jacques Cartier voyageait au 16ème siècle, nous remarquons que dans les contrées d'Europe occidentale, trois sortes au moins de calendrier sont en usage: le romain, le florentin, et le français. Dans le romain, l'année commençait comme aujourd'hui, le ler janvier, tandis que pour le florentin, elle débutait le 25 mars. Quant au système français, il faisait commencer son année avec le jour de Pâques. Pour compliquer davantage ce système, quelques provinces du sud de la France, comme la Guyenne, la Provence et quelques autres ne le suivaient pas; donc nous devons être prudent avec les documents de ces régions. ${ }^{2}$

Nous garderons pour Cartier les dates du calendrier français qui apparaissent dans le bref récit de ses voyages, ${ }^{3}$ et avec lui, nous allons faire la découverte d'Hochelaga. Est-ce à dire qu'aucun Blanc n'y soit venu avant ce navigateur né à Saint-Malo en 1491,4 et maître pilote de François 1er ? Nous ne saurions le dire dans l'état actuel de la documentation. ${ }^{5}$

* Travail présenté, lors de la réunion générale de l'Institut d'Histoire de l'Amérique française, le 30 avril 1966.

1 A titre d'exemple, voir la capitulation de Port-Royal, dans R.P. L. Le Jeune, Dictionnaire général... du Canada (2 vol., Université d'Ottawa, 1931).

2 Voir l'étude de l'abbé H.-A. Verreau, "Jacques Cartier: Questions de calendrier civil et ecclésiastique", Mémoires de la Société royale du Canada, section I.

3 Robert Le Blant, "Les écrits attribués à Jacques Cartier", Revue d'Histoire de l'Amérique française, XV (juin 1961): 90-103.

$4 \mathrm{Au}$ sujet de la date de naissance de Jacques Cartier, voir Jacques Cartier - Documents nouveaux, recueillis par Frédéric Joüon des Longrais (Paris, 1888), 5.

5 T. J. Oleson, Early Voyages and Northern Approaches, 1000-1632 (Toronto, 1963). 
Un fait demeure, Cartier fit dans sa jeunesse, avec des compatriotes, la pêche à la morue sur les bancs de Terre-Neuve. ${ }^{6}$ De plus, grâce aux expéditions qui le conduisirent sur les côtes des Amériques, il avait acquis une expérience exceptionnelle. ${ }^{7}$ Or, à cette époque, une idée hantait aussi bien les navigateurs que les souverains: trouver par le nord-ouest, le passage des Indes, terres de richesses réputées. ${ }^{8}$ Cartier avait pris officiellement possession de la Nouvelle France en 1534,9 le 24 juillet, mais il ne vint à Hochelaga que l'année suivante. Dans le récit de son voyage, il nous indique, notons-le bien, qu'il côtoya presque toujours la rive nord du Saint-Laurent, en quête du passage vers l'ouest. Aussi quand il partit de Stadaconé (i.e. Québec), le 19 septembre 1535, sur un galion de quarante tonneaux, l'Émérillon, ${ }^{10}$ sans guide indigène, il ne navigua que neuf jours, car les battures du lac St-Pierre l'empêchèrent de remonter plus loin. Alors Cartier confia au maître de ce galion, Jacques Maingart, assisté de plusieurs mariniers, la garde de ce bateau. ${ }^{11}$ Puis Jacques Cartier, accompagné de Claude de Pontbriand, échanson de Monseigneur le Dauphin; Charles de La Pommeraye; Jean Gouyon, seigneur de Thaumatz et du Marchais; Jean Poullet et vingt-huit mariniers montèrent partie dans la barque commandée par Macé Jalobert, partie dans celle aux ordres de Guillaume le Breton Bastille. Ces

6 Charles de La Morandière, Histoire de la pêche française de la morue dans l'Amérique septentrionale - Des origines à 1789 (2 vol., Paris, MCMLXII).

7 Charles de La Roncière, Jacques Cartier (Paris, 1947). - Paul Gaffarel, Les Français au delà des mers. Les Découvreurs français du XIVe au XVIe siècle. Côtes de Guinée, du Brésil et de l'Amérique du Nord (Paris, 1888).

8 Sur les idées de l'époque, voir Ternaux-Compans, Archives des Voyages; ou Collection d'anciennes relations inédites ou très rares, de lettres, mémoires, itinéraires et autres documents relatifs à la géographie et aux voyages ... (Paris, 1841).

9 H. P. Biggar, The Voyages of Jacques Cartier. Published from the originals with translations, notes and appendices (Ottawa, 1924), 64 . Publications of the Public Archives of Canada, no 11. Vraisemblablement sur le banc de sable appelé la Penouille dans la baie de Gaspé.

10 N.-E. Dionne, Jacques Cartier (Québec, 1889), 64.

$11 \mathrm{Sur}$ les compagnons de Cartier, voir N.-E. Dionne, ibid., 113-126 et 304-307. - Régis Roy, "Les compagnons de Cartier", Bulletin des recherches historiques (Lévis, 1919), XXV: 155-157. 
barques munies de voiles et de rames étaient en vue des îles de Sorel. ${ }^{12}$

Lisons le texte publié à Ottawa par H. P. Biggar en 1924 et intitulé: The Voyages of Jacques Cartier, ${ }^{13}$ pour en extraire quelques passages. Cartier et ses compagnons trouvèrent "cinq ou six belles ysles" puis ils allèrent à "l'vne d'icelles où trouvasmes cinq hommes qui prenoient des bestes sauvaiges, lesquelz vindrent aussi privement à noz barques, que s'ilz nous eussent veu toute leur vie, sans avoir peur ny craincte. Nous leur demandasmes par signes si c'estoit le chemyn de Hochelaga; et ilz nous monstrèrent que ouy et qu'il $\mathrm{y}$ avoyt encore trois journées à y aller." 14 Or de l'endroit où ils avaient laissé l'Émérillon, ils rencontrèrent sur leur trajet plusieurs Indiens qui leur "apportoient du poisson et autres victuailles, danssant et menant (montrant) grand joye de nostre venue. Et pour les attraire (attirer) et tenir en amytié avecq nous, leur donnoit le dict cappitaine (J. C.) pour recompance, des cousteaulx, pathenostres et aultres menues hardes, de quoy (ils) se contentoyent fort." 15 Rendu à Repentigny, Cartier longea les îles du Bout de l'Île et, pour être fidèle à son idée, il aurait dû prendre la rivière des Mille-Îles, mais à cet endroit, celle-ci coule presque à fleur de rochers, surtout à cette époque de l'année, "temps des plus petites eaues"; ${ }^{16}$ c'est pourquoi il s'engagea dans la Rivière des Prairies.

Les barques remontèrent donc cette rivière et le récit nous dit..." nous arryvez audict (pays de) Hochelaga, se randirent audavant de nous plus de mil personnes, tant hommes, femmes, que enffans, ... menant (montrant) vne joye merveilleuse; car les hommes en vne bande danssoient et les femmes de leur part, et les enffans $d\left[\right.$ e l'] aultre." ${ }^{17}$ Cartier descendit à terre avec

12 N.-E. Dionne, Jacques Cartier (Québec, 1889), 66.

13 H.P. Biggar, Publications of the Public Archives of Canada, no 11 (Ottawa, 1924). Tous les textes cités serviront à établir notre thèse et éviteront aux lecteurs le soin d'aller vérifier nos références.

14 H.P. Biggar, ibid., 147.

15 H.P. Biggar, ibid., 149.

16 H.P. Biggar, ibid., 146.

17 H.P. Biggar, ibid., 149-150. 


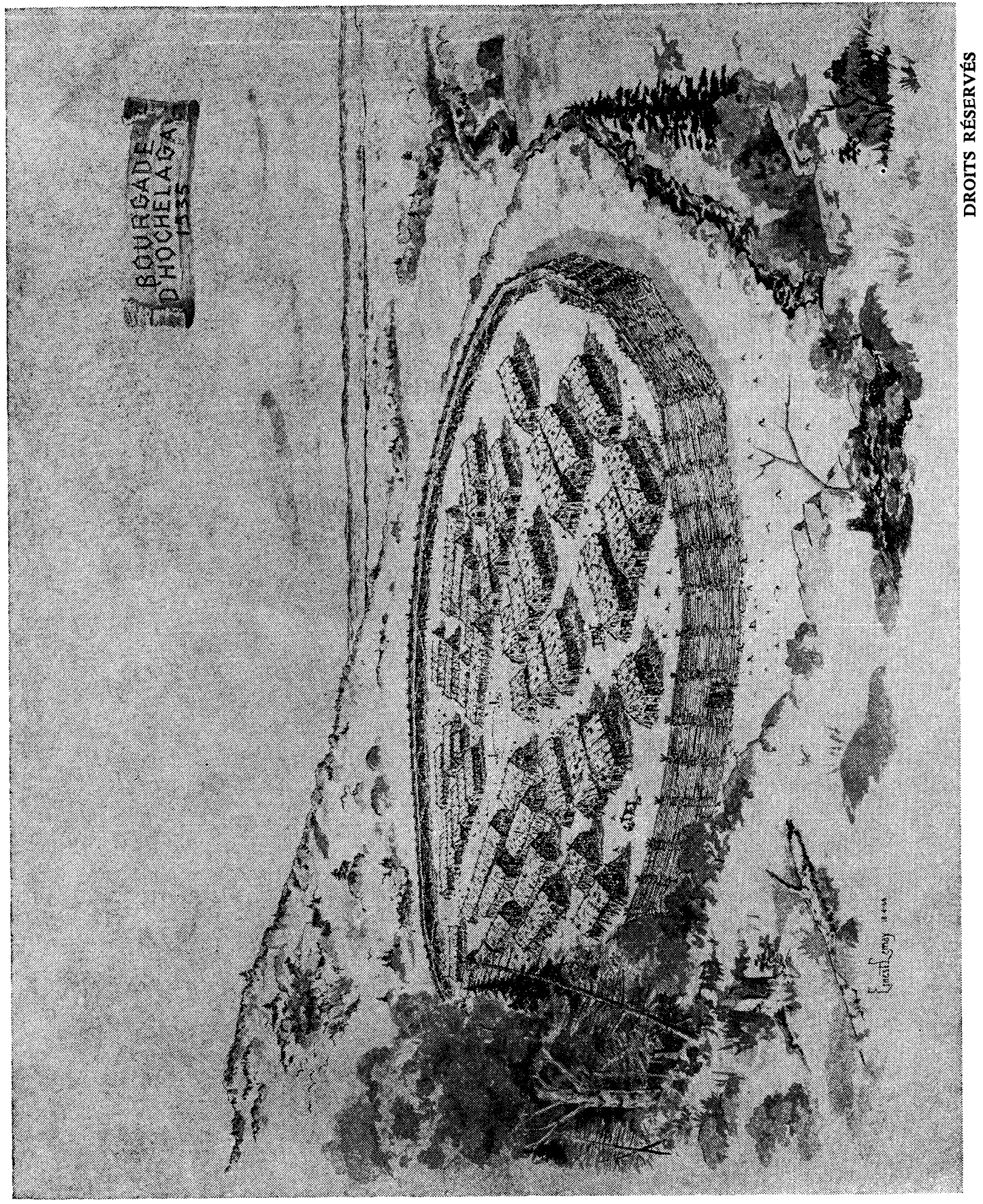


bon nombre de ses hommes et les Indiens les entourèrent et leur emmenèrent leurs femmes et leurs enfants afin de toucher au capitaine malouin. Que fit Cartier devant une telle démonstration d'amitié ? Nous voyons par le récit qu'il “... fict asseoir et ranger toutes les femmes, et leur donna certaines patenostres d'estaing, et aultres menues besongnes, (articles) et à partie des hommes des cousteaulx. Puis (il) se retira (avec ses hommes) à bort desdictes barques pour soupper et passer la nuict; durant laquelle demoura icelluy peuple sus le bort dudict fleuve, au plus près desdictes barques, faisant toute (la) nuict plusieurs feuz et dansses, en disant à toutes heures, aguyase, qui est leur dire de salut et joye." 18

\section{C'était samedi, le deux octobre 1535}

Il est à présumer que la première nuit passée par Jacques Cartier et ses hommes ne fut pas d'un grand repos, à cause des danses, des manifestations et des cris de joie des Indiens qui ponctuaient leurs réjouissances du mot "aguyase" à intervalles réguliers. C'est pourquoi, "Le landemain, au plus matin, le cappitaine (J. C.) se acoustra (vêtit son costume d'apparat), et fict mectre (mettre) ses gens en ordre, pour aller veoyr la ville et demourance (demeure) dudict peuple, et vne montaigne, qui est jacente à ladicte ville, où allèrent avecques ledict cappitaine les gentilz-hommes et vingt mariniers, et laissa le parsus (le surplus) pour la garde des barques; et print (prit) troys hommes de ladicte ville de Hochelaga, pour les mener et conduyre audict lieu." 19

Comment apparut à Jacques Cartier le chemin pour se rendre à Hochelaga ? Laissons-lui la parole: "Et nous estans en chemin, le trouvasmes aussi battu qu'il soit possible de veoyr, et la plus belle terre, et meilleure [qu'on sçauroit veoir, toute] plaine de chaisnes (chênes), aussi beaulx qu'il y ait en forestz de France, soubz lesquelz estoit toute la terre couverte

18 H.P. Biggar, ibid., 151.

19 H.P. Biggar, ibid., 152-153. 
de glan." 20 La marche vers Hochelaga fut marquée d'un incident: la rencontre d'un chef indien. Pour savoir ce qui s'y passa, nous allons consulter le récit de Cartier: "Et nous aians [marché] envyron (une) lieue et demye, trouvasmes sur le chemin l'vn des principaulx seigneurs de ladicte ville de Hochelaga, avecq plusieurs personnes, lequel nous fict signe qu'il se failloit reposer audict lieu, près vng (un) feu qu'ilz avoient faict audict chemin; [ce que feismes]. Et lors commança ledict seigneur à faire vng sermon et preschement, comme cy davant est dict, estre leur coustume de faire joie et congnoissance, en faisant celluy seigneur chère audict cappitaine et sa compaignie" ${ }^{21}$ (i.e. en leur souhaitant la bienvenue). Évidemment ce discours fut presque incompréhensible à ces européens, aussi Cartier remercia ce chef indien de la façon suivante: "Lequel cappitaine (J. C.) luy donna vne couple de haches et vne couple de cousteaulx, avec vne croix et remembrance de cruxifix qu'i[1] luy fit baiser et la luy pendit au col; de quoy (le chef) rendit graces audict cappitaine." ${ }^{22}$ Cette pause terminée, Cartier, sa troupe et ses trois guides indiens se remirent en marche et voici la description des lieux: "Ce faict, (nous) marchames plus oultre, et envyron (une) demye lieue de là, commançasmes à trouver les terres labourées et belles, grandes champaignes (campagnes), plaines de bledz de leur terre, qui est comme mil du Brezil, aussi groz, ou plus, que poix, duquel (ils) vivent, ainsi que nous faisons du froument. Et au parmy (milieu) d'icelles champaignes, est scitué[e] et assise ladicte ville de Hochelaga, près et joignant vne montaigne, qui est, à l'entour d'icelle..." 23

Quel est l'aspect extérieur de cette ville ? Cartier nous le dit: "Ladicte ville est toute ronde, et cloze de boys, (bois) à troys rancqs, (rangs) en façon d'vn[e] piramyde, croizée par le hault, ayant la rangée du parmy (milieu) en façon de ligne perpendicullaire; puys (une) rangée de boys couchez de long,

20 H.P. Biggar, ibid., 153.

21 H.P. Biggar, ibid., 153-154.

22 H.P. Biggar, ibid., 154.

23 H.P. Biggar, ibid., 154-155. 
bien joings et couzus à leur modde; et est de la haulteur d'envyron deux lances" 24 (Une lance du 16ème siècle mesurait plus de quinze pieds). "Et n'y a en icelle ville qu'vne porte et entrée, qui ferme à barres, sur laquelle, et en plusieurs endroitz de ladicte closture, y a manières de galleryes, et eschelles à y monster, lesquelles sont garnies de rochyers et cailloux, pour la garde et deffence d'icelle." ${ }^{25}$ Nous allons pénétrer dans cette ville pour être à même de juger non seulement de sa grandeur mais encore de ses maisons. Afin de ne rien inventer, nous citerons toujours Cartier: "Et par noz guides et conducteurs fumes menez au meilleu d'icelle ville, où il $\mathrm{y}$ a vne place entre les maisons, spacieuse d'vn gect (jet) de pierre, en carré ou envyron, lesquelz (indiens) nous firent signe que nous arrastacions audict lieu, ce que (nous) fymes." 26 Que se passa-t-il alors ? "Et tout soubdain, s'assemblèrent toutes les femmes et filles de ladicte ville, dont l'vne partie estoient chargées d'enffans entre leurs braz, qui nous vindrent [frotter] le visaige, braz et aultres endroiz de dessus le corps, ou ilz pouvoient toucher, pleurant de joye de nous veoyr, nous faisant la meilleur [e] chère (accueil) qu'il leur estoit possible, en nous faisant signes qu'il nous pleust toucher leursdictz enffans. Après ces choses faictes, les hommes firent retirer les femmes, et se assirent sus la terre, à l'entour de nous, comme si (nous) eussions voullu jouer vng mistère (pièce de théâtre). Et tout incontinent revindrent plusieurs femmes, qui apportèrent chascune une natte carrée, en façon de tapisserye, et les estandirent sus la terre, au meilleu de ladicte place, et nous firent mectre sus icelles. Après lesquelles choses ainsi faictes, fut apporté, par neuf ou dix hommes, le Roy et seigneur du pays, qu'ilz appellent en leur langue agouhanna, lequel estoit assis sus vne grande peau de serf; et le vindrent poser dedans ladicte place, sus lesdictes nattes, [au]près du cappitaine, en [nous] faisant signe que c'estoit leur [Roy et] seigneur." ${ }^{27}$

24 H.P. Biggar, ibid., 155.

25 H.P. Biggar, ibid., 156.

26 H.P. Biggar, ibid., 162.

27 H.P. Biggar, ibid., 162-164. 
Pour se faire une idée de ce grand chef indien, Cartier nous en a laissé un bon portrait: "Celluy agouhanna estoit de l'aige d'envyron cinquante ans, et n'estoit poinct mieulx acoustré que les aultres, fors qu'il avoyt, à l'entour de sa teste, vne manière de lizière rouge, pour sa couronne, faicte de poil $d[e]$ herissons; et estoit celluy seigneur tout percludz et malade de ses membres. Après qu'il eut faict son signe de salut audict cappitaine et à ses gens, en leur faisant signes évidans qu'ilz fussent les [très] bien venuz, il monstra ses braz et [ses] jambes audict cappitaine, luy faisant signe qu'il luy pleust les toucher, comme s'il luy eust demandé garison et santé." ${ }^{28}$ Devant une situation pareille, Cartier jugea qu'il n'avait qu'une chose à faire: "Et lors le cappitaine commança à luy frotter les braz et jambes, avecq les mains. Et [lors] print ledict agouhanna la lizière et couronne qu'il avoit sus la teste, et la donna audict cappitaine. Et tout incontinent, furent amenez audict cappitaine plusieurs malades, comme aveugles, bourgnes, boisteulx, impotens, et gens si très-vieulx que les paupières des yeulx leurs pendoient [jusques] sus les jouez, les seoyant et couchant près ledict cappitaine pour les toucher, tellement qu'il sembloyt que Dieu fust là descendu, pour les gueryr." ${ }^{29}$ Devant une scène si émouvante, Cartier, en chrétien qu'il était, se sentit bouleversé. La suite de son récit nous le montre bien: "Ledict cappitaine, voyant la pitié et foy de cedict peuple, dist l'euvangille (de) sainct Jehan, sçavoir: l'Im principio, faisant le signe de la croix sus les pauvres malades, priant Dieu qu'il leur donnast congnoissance de nostre saincte foy, et de la passion de Nostre Saulveur, et grace de recouvre[r] chrestienté et baptesme. Puis print ledict cappitaine vne paire d'heures (deux heures), et tout haultement leut, (lut) de mot à mot, la passion de Nostre Seigneur, si (bien) que tous les assistants la peurent ouyr, où tout ce pouvre peuple fist vne (sic) grande sillance, et furent merveilleusement bien entendibles, regardant le ciel et faisant pareilles serimonyes qu'ilz nous veoyent faire." 30

28 H.P. Biggar, ibid., 164.

29 H.P. Biggar, ibid., 164-165.

30 H.P. Biggar, ibid., 165-166. 
Cartier qui avait acquis une certaine expérience du comportement des Indiens termina cette rencontre de la façon suivante: "Après laquelle, (il) fist ledict cappitaine ranger tous les hommes d'vn cousté (côté), les femmes d'vn aultre, et les enffans d'aultre, et donna aux principaulx des hachotz, es (aux) aultres des cousteaulx, et es femmes des patenostres et aultres menues choses; puis gecta (jeta) parmy la place, entre lesdictz [petis] enffans, des petites bagues et agnus Dei d'estaing; de quoy (ils) menèrent (montrèrent) vne merveilleuse joye. Ce faict, le cappitaine commanda (de) sonner les trompettes et aultres instrumens de musicque, de quoy ledict peuple fut fort resiouy." 31

Pierre Marquier fut ainsi le premier trompettiste à jouer à Montréal ${ }^{32}$

Avant de quitter Hochelaga, Cartier a tenu à nous donner des détails intéressants sur le logement des Indiens ainsi que sur leur manière de vivre. Voici ce que nous lisons à ce propos: "Il y a dedans icelle ville envyron cinquante maisons, longues de envyron cinquante pas ou plus, chascune, et douze ou quinze pas de laize (large), toutes faictes de boys, couvertes et garnyes de grandes escorces et pellures desdictz boys, aussi larges que tables, bien cousues artifficiellement, selon leur modde. Et par dedans icelles y a plusieurs aistres et chambres; et au meilleu (milieu) d'icelles maisons, y a vne grande salle par terre, où [ilz] font leur feu, et vivent en communaulté; puis se retirent en leursdictes chambres, les hommes avecq leurs femmes et enffans. Et pareillement, [ilz] ont greniers au hault de leurs maisons, où [ils] mectent leur bled, duquel ilz font leur pain, qu'ilz appellent carraconny..." 33 Cartier nous donne aussi une idée sur la manière qu'ont les Indiens de se faire à manger, de se coucher, de se vêtir et en général de vivre.

31 H.P. Biggar, ibid., 166-167.

32 N.-E. Dionne, Jacques Cartier (Québec, 1889), 306. Dans le rôle de compagnons de Cartier, Pierre Marquier est le seul qui y figure comme "trompette".

33 H.P. Biggar, ibid., 156-157. 
Mais on peut se demander comment entre eux se faisaient les échanges et qu'est-ce qui leur servait de monnaie ? $\AA$ première vue, on serait porté à croire que les peaux de fourrures remplissaient cette office, mais tel n'est apparemment pas le cas. En effet, Cartier nous dit: "La plus precieuse chose qu'ilz ayent en ce monde est esnoguy (coquillage), lequel est blanc comme neif (neige), et le prennent audict fleuve en cornibotz (semblable à un cornet à bouquin), en la manière qui ensuyt." Et dit Cartier, de ce (esnoguy) usent, comme nous faisons d'or et d'argent." ${ }^{34}$ Pour se procurer ces coquillages, les Indiens se servaient d'un cadavre frais que l'on a tailladé par tout le corps et que l'on plongeait dans l'eau pendant une douzaine d'heures à un endroit bien déterminé. Puis ils le retiraient et quantité d'esnoguy adhérait aux endroits tailladés. Ce qui rendait ce coquillage précieux, c'était la vertu ou la propriété qu'il avait d'étancher le sang. D'ailleurs Cartier en fit l'expérience et voici ce qu'il en dit: "Il (esnoguy) a la vertu d'estancher le sang des nazilles; car nous l'avons expérimenté." 35

Quelle était l'importance d'Hochelaga par rapport aux autres tribus indiennes du Saint-Laurent? Cartier nous en a fourni la réponse: “... ilz ne bougent de leur pays, et ne sont embulataires (nomades), comme ceulx de Canada et du Saguenay; non obstant que lesdictz Canadians leurs soient subgectz, avec vIII ou IX aultres peuples qui sont sur ledict fleuve." 36 Une fois la visite d'Hochelaga terminée, Cartier nous dit: "Après que nous fumes sortis de ladicte ville, fumes conduictz par plusieurs hommes et femmes d'icelle sur la montaigne [cy] davant dicte, qui est par nous nommée mont Royal, distant dudict lieu d'vn cart de lieue." 37 Que fit Cartier sur la montagne et que vit-il ? "Et nous estans sus ladicte montaigne, eusmes veue et congnoissance de plus de trente lieues, à l'envyron d'icelle; dont il y a, vers le nort, vne rangée de montaignes, qui sont est et ouaist gisantes, et autant devers le su. Entre lesquelles montaignes

34 H.P. Biggar, ibid., 159-160.

35 H.P. Biggar, ibid., 161.

36 H.P. Biggar, ibid., 161.

37 H.P. Biggar, ibid., 168. 
est la terre, la plus belle qu'il soit possible de veoyr, labourable, vnye et plaine. Et par le meilleu (milieu) desdictes terres, voyons ledict fleuve oultre le lieu où estoient demourées noz barques, où il y a vng sault d'eaue, le plus impetueulx qu'il soit possible de veoir, lequel ne nous fut possible de passer; et voyons icelluy fleuve tant que l'on pouvoyt regarde[r], grand, large et spacieulx, qui alloit au surouaist, et passoit par auprès de troys belles montaignes rondes, que nous voyons, et estimyons qu'elles estoient à envyron quinze lieues de nous. Et nous fut dict et monstré par signes, par les troys hommes [du pais] qui nous avoyent conduictz, qu'il y avoyt troys ytieulx saultz d'eaue audict fleuve, comme celluy où estoient nosdictes barques; mays nous ne peusmes entendre quelle distance il y avoyt entre l'vn et l'aultre, [par faulte de langue]." 38 Nous pouvons supposer qu'après une telle journée d'exploration, Cartier et ses hommes, en armes et avec leurs instruments de musique, devaient avoir hâte de retourner à leurs barques vu la fatigue et la faim, car ils n'avaient pas mangé des mets indiens à cause dit Cartier que: "Tout leur vivre est sans aucun goust de sel." ${ }^{39}$

Que dit le récit de voyage quant au retour de cette expédition et quel fut le comportement des Indiens envers ces Blancs ? "Après lesquelles choses ainsi veues et entendues, nous retirasmes à noz barques, qui ne fut sans avoir conduicte du grand numbre dudict peuple, dont partie d'eulx, quand (ils veoyoient noz gens laz (fatigués), les chargeoient sus eulx, comme sus chevaulx, et les portoyent. Et nous arrivez à nosdictes barques, fimes voille pour retourner à nostre gallion, pour doubte qu'ils n'eust aucun encombrier. Lequel partement (départ) ne fut (pas) sans grand regret dudict peuple; car tant qu'ilz nous peurent suyvir aval ledict fleuve, ilz nous suyvèrent. Et tant fismes, que nous arrivasmes à nostredict gallion le lundi, quatriesme jour d'octobre. Le mardi, cinquiesme jour dudict moys, nous fismes voille et appareillasmes avecq nostredict gallion et

38 H.P. Biggar, ibid., 168-169.

39 H.P. Biggar, ibid., 159. 
barques, pour retourner à la prouvince de Canada, au port de sainte Croix, où estoient demourez nosdictz navires. ${ }^{40 "}$

Si nous avons cité tous ces textes, c'est afin de pouvoir en tirer des conclusions. Cependant nous aurons besoin d'un aperçu d'un autre voyage de Cartier ici en 1541, ainsi que des lettres de son neveu Jacques Noël pour établir, grâce à ces textes, ainsi qu'à la carte harléienne faite circa 1536 et à l'aide d'autres récits de voyages, l'endroit de débarquement de Cartier à Montréal, le chemin parcouru jusqu'à la halte qui lui fut imposée par la rencontre d'un chef indien, la localisation d'Hochelaga, et le point où Cartier a été sur la montagne. Nous avons vu qu'en 1535, Jacques Cartier s'était arrêté au "premier sault" parce que celui-ci était infranchissable. Il avait appris aussi par les Indiens qu'il y en avait trois, mais que par "faute de langue", il ne put en estimer la distance entre chacun. C'est pourquoi, à son voyage de 1541, Cartier voulut poursuivre son exploration et tenter de franchir ces trois "saultz". Voyons comment aboutit cette tentative, puisqu'il était parti de CharlesbourgRoyal, près de Québec, le 7 septembre 1541, avec quelques gentilshommes, dont Martin de Paimpont et un bon nombre de mariniers divisés en deux barques munies de voiles et de rames. ${ }^{41}$ Avant de vous donner ce récit du voyage de 1541 , notons qu'il ne nous est parvenu que par la traduction italienne de Ramusio. ${ }^{42}$ M. H. P. Biggar l'a publié en anglais et nous l'avons traduit en français dans le langage de Cartier. Donc le récit nous dit:

"Et nous navigasmes avecq si bon vent, que nous arrivasmes le onziesme jour du moys (à Montréal) au premier sault d'eaue, lequel est à deulx lieues de distance de la ville de Tutonaguy. Et après que nous fumes arrivez là, (au premier sault) nous décidasmes d'aller plus oultre et de remonter aussy loin que ce fust possible avecq vne des barques, pendant que l'aultre resteroit là jusques à son retour; et nous doublasmes le nombre des rameurs pour remonter le courant dudict sault. Et après

40 H.P. Biggar, ibid., 172.

41 H.P. Biggar, ibid., 256.

42 H.P. Biggar, ibid., introduction, xii. 
que nous eusmes franchi vne partie du chemyn de notre aultre barque, nous trouvasmes mauvais fonds et grosses roches, et vng courant si fort, qu'il nous fust pas possible d'aller plus loing avecq notre barque, et le cappitaine résolut d'aller par terre pour veoyr la nature et façon du sault 43 " (texte traduit de l'anglais en langage de Cartier par le soussigné).

Ce qui découle de ce texte est donc clair, Cartier n'a pu franchir le "premier sault" pour se rendre au second, même en doublant les rameurs. En conséquence, pour l'atteindre, il lui fallut prendre le chemin de terre. Comment se fit cette expédition? Consultons le récit.

"Et après que nous fusmes arrivez sus le rivage, nous trouvasmes près de la rive vng chemyn et sentier battu allant vers lesdictz saultz, vers lesquels nous nous dirigeasmes. Et sus ledict chemyn et peu après, nous trouvasmes une habitation de gens lesquelz nous firent grande chère (accueil) et nous traitèrent très amicalement. Et après qu'il (Cartier) leur eust signifié que nous allyons vers les saultz et que nous désiryons aller au Saguenay, quatre jeunes hommes vindrent avecq nous pour nous monstrer le chemyn, et ilz nous menèrent si loing que nous arrivasmes à vng aultre village ou demourance de bonne gens, lesquelz demouroient au dessus et près du second sault, et lesquelz vindrent et nous apportèrent de leurs victuailles, comme pottage et poissons, et nous en offrirent. Après que le cappitaine se fust informé auprès d'eulx aussy bien par signes que par paroles, combien il y avoyt encore de saultz nous avyons à passer pour aller à Saguenay, et qu'elle distance et chemyn il $\mathrm{y}$ avoyt jusque là, ce peuple nous monstra et nous donna à entendre que nous estyons au second sault et que de là, il n'y en avoyt qu'vng aultre à passer que le fleuve n'estoit pas navigable pour aller à Saguenay et que ledict sault n'estoit qu'à une tierce partie de plus que (ce que) nous avyons parcourue, nous monstrans icelluy avecq certains petits bastons, lesquelz ilz placèrent sur la terre à vne certaine distance et ensuite posèrent d'aultres petites branches entre les deulx, représentans

${ }^{43}$ H.P. Biggar, ibid., 257, texte anglais. 
les saultz. Et par lesdictez marques, si leurs dictz sont vraiz, il ne peut y avoir que six lieues par terre pour passer lesdictz saultz" 44 (texte traduit de l'anglais par le soussigné).

Il est important de noter ici que Cartier a fait un estimé des distances d'après les données indiennes et non pour les avoir parcourues entièrement. C'est pourquoi, il ne faut pas tenir grief au capitaine Malouin, si les mesures ne sont pas rigoureusement exactes avec celles que nous connaissons aujourd'hui. Il faut ajouter aussi que non seulement chaque province de France, mais pratiquement chaque ville avait son système de mesures. ${ }^{45}$ C'est une autre raison qui expliquerait l'appréciation de Cartier. Enfin, celui-ci nous dit pourquoi il n'a pu vérifier lui-même toutes ces distances.

"Après que nous fusmes avertiz par ledict peuple de ces choses cy dessus mentionnéez et aussy parce que le jour estoit bien avancé et que nous n'avyons ny bu ny mangé de cette journée, nous décidasmes de retourner à nos barques, et nous vinmes là où nous trouvasmes vng grand numbre de gens s'élevans à 400 personnes ou envyron, lesquelz sembloyent nous faire très grande chère (accueil) et se resiouyr de nostre arrivée" ${ }^{46}$ (texte traduit par le soussigné).

Pour se garder l'amitié des Indiens, Cartier devant pareille démonstration, comprit qu'il devait se comporter en grand seigneur, nous le constatons par le récit:

"En conséquence nostre cappitaine donna à chacun d'eulx certaines petites besongnes (articles), comme peignes, espingles d'estaing et de laiton et aultres menues pacotilles et à chacun des chefs sa hachette et son hameçon; sur quoi ilz poussèrent des cris et firent des serimonyes de joye ${ }^{47}$ " (texte traduit par le soussigné).

44 H.P. Biggar, ibid., 257-258, texte anglais.

45 M. Gournay, Tableau général du commerce (Paris, 1789-90), 729.

46 H.P. Biggar, ibid., 258, texte anglais.

47 H.P. Biggar, ibid., 258-259, texte anglais. 
Après cette distribution de présents, le récit nous dit: "Cela faict, nous retournasmes avecq noz barques..." (texte traduit par le soussigné).

Maintenant que nous connaissons les textes importants de Cartier au sujet de ces deux voyages à Montréal, quelles sont les conclusions qu'il faut en tirer ?

1 - Cartier aussi bien en 1535 qu'en 1541 n'a pu franchir un "sault" qu'il dit être le "premier sault".

2 - Cartier en 1535, a appris l'existence de "troys saultz", mais ne put en déterminer la distance entre chacun par "faute de langue", tandis qu'en 1541, il va par chemin de terre du "premier au second sault". Il nous donne par la même occasion les raisons qui l'ont empêché de se rendre à un troisième et dernier "sault".

3 - Le capitaine malouin s'est fait expliquer par des Indiens qui vivaient au dessus du second "sault", grâce à des petits bâtons placés sur le sol, que le chemin à parcourir pour atteindre le troisième "sault" était égal au tiers de la distance qu'il avait marché entre le premier et second "sault". Il en a donc déduit, si les Indiens ont dit la vérité, qu'entre le premier et troisième "sault", par chemin de terre, notons-le bien, il y avait six lieues. D'où entre les deux premiers, il a marché quatre lieues et demie.

4 - $\AA$ propos de ces trois "saults", essayons de les situer, soit sur le St-Laurent, soit sur la rivière des Prairies.

\section{A - SUR LE ST.LAURENT}

Voyons s'il peut y avoir trois "saults", dont le premier est infranchissable. Si l'on prend le "Courant Ste-Marie" comme premier "sault", c'est une fausseté parce qu'on le franchissait aussi bien sous le régime français ${ }^{49}$ que sous le régime anglais. ${ }^{49 a}$

48 H.P. Biggar, ibid., 259, texte anglais.

49 Dollier de Casson, Histoire de Montréal - A History of Montreal 1640-1672 from the french of Dollier de Casson, translated and edited, with a life of the authors, by Ralph Flenley (London and Toronto, 1928).

49a William Henry Atherton, Montreal 1535-1914 under British Rule 1760-1914 (Montreal, 1914). 
Si on reporte le premier "sault" aux rapides de Lachine, où situer les deux autres d'après les données de Cartier ? Il est donc évident que ce n'est pas par le fleuve St-Laurent que Cartier est venu à Montréal.

\section{B - SUR LA RIVIÊRE DES PRAIRIES 50}

Est-ce qu'il y a trois "saults" ou rapides sur cette rivière ? On peut le constater facilement, puisqu'on a celui du Sault-auRécollet, celui du Cheval-Blanc et celui du Hollandais que certains appellent l'Allemand. D'ailleurs une carte hydrographique de la rivière des Prairies, faite en 1908 par les ingénieurs du gouvernement du Canada, donc avant la construction des barrages pour les pouvoirs électriques, nous donne les différents niveaux d'eau tout le long de cette rivière et indique les différents "saults", ou rapides mentionnés plus haut. En effet de l'île de la Visitation, on a une élévation moyenne de trente pieds, il en est de même au Pont-Viau, tandis qu'à l'île Perry on a 43 pieds et au rapide du Cheval-Blanc $53^{\prime}$ et $57^{\prime}$ à celui du Hollandais. ${ }^{51}$

5 - La carte harléienne faite circa $1536,,^{52}$ nous montre que la "ville" d'Hochelaga est située du côté sud du premier "sault". Or si Cartier était arrivé à Montréal par le fleuve St-Laurent, Hochelaga aurait été situé du côté sud de celui-ci, ce qui est̂ incompatible avec le texte de Cartier puisque ce dernier nous

50 Nous avons déjà, dans la revue Bonsecours, no 5, IX: 21, du mois de décembre 1965, signalé la contribution de M. Aristide BeaugrandChampagne, dans le Cahier des Dix, no 12 (1947): 115 . . comme l'un des premiers auteurs à soutenir que Jacques Cartier était arrivé par la rivière des Prairies. Nous sommes d'accord sur ce point et presque entièrement sur celui du diamètre de la bourgade. Ce sont là, les deux points que nous avons en commun.

51 Carte côtée 4A, Public Works Canada Georgian Bay Ship Canal Lake Huron to Montreal 22 foot Channel Projet Riviere des Prairies, Alternative Route, 1908.

52 H.P. Biggar, The Voyages of Jacques Cartier (Ottawa, 1924), entre les pages 128-129. Notons que cette carte est publiée d'après l'original et donnée comme étant circa 1536. Marcel Trudel, Atlas historique $d u$ Canada français (Les Presses de l'Université Laval, 1961), 18, indique la date de circa 1542, tandis que Lionel Groulx, La découverte du Canada - Jacques Cartier (Montréal, Fides, 1966), 130, reproduit la carte harléienne de Desceliers en 1546. 
dit que la "ville" d'Hochelaga est adjacente à une montagne qu'il nomma Mont-Royal.

6 - Le Père Dalmas, s.j., dans son récit de voyage sur la rivière des Prairies, le 25 septembre 1674, avec Charles Lemoyne et Pierre Gagnié, les deux meilleurs "canoteurs" de la région, mentionne cinq "saults" ou rapides dont le courant "est très Impétueux en quelques-uns". ${ }^{53}$ Comme Cartier ne fait mention que de "troys saultz", on doit en déduire ceci, ou bien il n'y en avait que trois à son époque, ou bien s'il y en avait cinq, deux de ceux-ci ne comportaient pas de difficultés particulières pour les franchir, et ce serait pourquoi il n'en parle pas.

7 - Arrivé par la rivière des Prairies en 1535 et en 1541, où Cartier est-il débarqué ? C'est ce que nous allons chercher à déterminer, grâce à différentes sources de renseignements.

7-A - Le texte de Cartier nous dit que ce fut au premier "sault" infranchissable. Grâce aux distances qu'il nous donne entre les "saults" ainsi qu'entre son point de débarquement et la ville d'Hochelaga et le Mont-Royal, il est possible de délimiter l'endroit à l'aide des accidents géographiques de la rivière des Prairies.

1 - $\mathrm{Au}$ ruisseau Vinet, c'est-à-dire vis-à-vis la rue Curotte, du côté ouest de l'église du Sault-au-Récollet ?

2 - Au ruisseau Provost, c'est-à-dire vis-à-vis la rue Meunier, à l'est de l'hôpital de Notre-Dame de la Merci ?

3 - Au ruisseau Raimbault, c'est-à-dire vis-à-vis le boulevard St-Germain, à l'est de l'hôpital du Sacré-Cœur ?

4 - $\mathrm{Ou}$ ailleurs sur la rivière des Prairies ?

7-B - Le voyage du Père Dalmas, s.j. va nous aider puisqu'il a été fait presque à la même époque de l'année que celui de Cartier, i.e. en automne. Voici ce qu'il dit: “... sur les 9 ou dix heures (du matin le 25 septembre 1674), nous montasmes la

53 Reuben Gold Thwaites, The Jesuit Relations and Allied Documents (72 vol., Cleveland, 1899), LVIII : 112. 


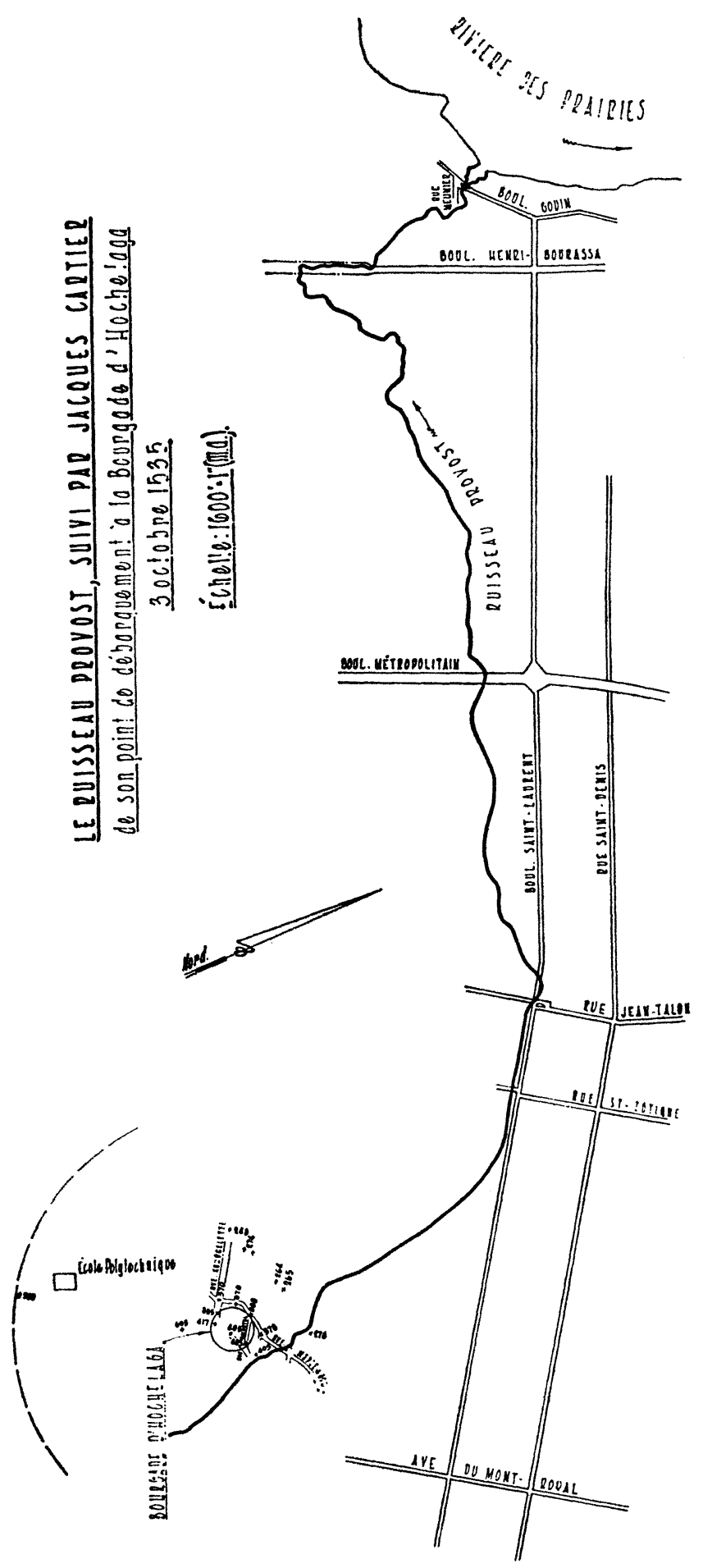


Riviere des prayries et Cabannasmes au dessus du troisiesme rapide". ${ }^{54}$ On remarque de suite que le premier et deuxième rapide ne lui ont pas causé de difficultés particulières; notons ici que c'est peut-être la raison que Cartier avait pour n'en point parler s'ils existaient dans son temps. Le texte de ce Jésuite nous situe le troisième rapide, voici: “... et le troisiesme proche d'vne petite Jsloye ou est la force du courant qui donne bien de la peine a ceux qui traisnent quoiquils se tiennent a cette Jsloye le 3e. rapide est au dessus du 2e. vne lieüe ou enuiron (environ)". ${ }^{55}$

7-C - La carte du voyage du Père Dalmas, s.j., ${ }^{56}$ nous montre qu'il cabana avec ses "canoteurs" au dessus du $3 e$ "sault" et l'on peut voir une petite île sur le côté de Montréal où le courant est tellement fort qu'il est impossible même en canot de le remonter. Il faut absolument y faire un portage. Est-il nécessaire d'ajouter un autre témoignage pour montrer les difficultés de Cartier? Si nous consultons Pierre Boucher dans son Histoire véritable et naturelle, au sujet de la rivière des Prairies, voici ce qu'il dit: "Je ne feray point la description des Terres qui se rencontrent des deux costez de cette riuiere qui tire au Nort, veu qu'il est mal-aisé d'y pouuoir habiter à cause des sauts ou cascades d'eaux qui s'y rencontrent, qui empeschent la riuiere d'estre nauigable à d'autres bastimens qu'aux petits Vaisseaux dont se seruent nos Sauvages, qui peuuent estre transportez d'vn lieu a vn autre, sans autre machine que les épaules d'vn hôme ou de deux au plus." 57 Alors, imaginez les barques de Cartier, ce qu'elles ont pu faire devant l'impétuosité du courant. C'est pourquoi Cartier nous dit qu'il est infranchissable.

7-D — La carte de Montréal, faite en $1702^{58}$ et attribuée

54 Thwaites, ibid., 116.

55 Thwaites, ibid., 118.

56 Carte conservée au Séminaire de Québec.

57 Pierre Boucher, Histoire véritable et naturelle des mœurs et production du pays de la Nouvelle-France vulgairement dite le Canada 1664 (éd. de la Société historique de Boucherville, 1964), 36-37.

58 Carte conservée au Séminaire de Saint-Sulpice, 6 rue du Regard, Paris. M. Irénée Noye, archiviste. 
à M. de Belmont, nous situe le Sault-au-Récollet vis-à-vis l'île Raisin, c'est-à-dire à l'endroit où les Sulpiciens établirent un moulin en 1798 appelé le moulin du "Gros Sault". Cette île Raisin correspond à celle désignée par le Père Dalmas comme la "petite Jsloye" du troisième rapide, donc un peu à l'ouest du ruisseau Provost qui forme une baie à cet endroit où les barques de Cartier pouvaient se mettre à l'abri du courant.

7-E - En 1797, Louis Guy, ${ }^{59}$ arpenteur, prépara un plan de la terre concédée en 1728 à Mlle Louise De Couagne. Nous y voyons l'île Raisin et la presqu'île située du côté est de cette île. Dans ce contrat de concession, les seigneurs de Montréal avaient déjà prévu la construction d'un moulin sur cette presqu'île, c'est pourquoi ils s'étaient réservé le droit de racheter cet emplacement. Aussi quand ils construisirent leur moulin en 1798, ils firent creuser un canal à travers cette presqu'île et unirent l'île Raisin à celle-ci. Quand M. Charles Perry acheta ce moulin le 19 juillet 1837, ${ }^{60}$ tout l'emplacement prit le nom d'île Perry qu'il a conservé jusqu'à nos jours.

7-F — La carte hydrographique de 1908 citée plus haut, nous montre grâce aux différences de niveaux d'eau que Cartier ne pouvait aller plus loin que l'île appelé aujourd'hui Perry.

7-G - D'après l'expérience de deux canoteurs contemporains, Messieurs Claude et Marcel Giroux de Cartierville, qui ont fait le trajet sur la rivière des Prairies en 1927, avec deux compagnons, pour se rendre en canots jusqu'à Nicolet, aller et retour, selon les témoignages que j'ai recueillis d'eux, le plus loin que pouvait se rendre Cartier était au ruisseau Provost, non seulement à cause de l'impétuosité du courant, mais aussi à cause du genre de construction des barques du capitaine malouin. En effet, celles-ci devaient avoir un tirant d'eau de deux à trois pieds. Donc il était impossible pour Cartier rendu à l'île Perry de franchir le reste du Sault-au-Récollet.

59 Plan de Louis Guy, dans Robert Prévost, Le Moulin du Gros-Sault (Editions Archonte, Montréal, s.d.), 27.

60 Robert Prévost, ibid., 61. 
7-H - Il ne faut pas oublier que Cartier, de la montagne, vit le lieu où étaient demeurées ses barques "où (précise-t-il) il y a vng sault d'eaue, le plus impetueulx qu'il soit possible de... passer".61 Donc au même endroit où le Père Dalmas nous dit qu'il est impossible de franchir ce courant.

7-I - Est-ce que Cartier serait allé du côté du Sault SaintLouis, comme le mentionnent Champlain, le Père Denys Jamet, Sixte Le Tac, les Relations des Jésuites, etc. ? Peut-être, mais nous n'avons aucune preuve documentaire puisqu'une partie du manuscrit de Cartier pour l'année 1541 est perdue et que l'autre partie ne nous est parvenue que par une traduction italienne. ${ }^{62}$ Si ce manuscrit ne l'était pas du temps de ces auteurs-là, malheureusement pour nous, ils ne le citent pas, donc nous ignorons où ils peuvent avoir obtenu ce renseignement, à moins de l'avoir pris sur la carte de Desceliers de 1550 qui dit: "Jusqu'ici (Sault Saint-Louis) monta M. de Roberval." 63 Or il ne faut pas oublier que lorsque Roberval vint au Canada, Cartier était déjà sur le chemin du retour. Après une brève escale à St-Jean, TerreNeuve, où ils furent en désaccord, Jacques Cartier partit de nuit pour la France. ${ }^{64}$

7-J - Nous devons donc conclure, dans l'état actuel de la documentation, que le point de débarquement de Cartier se situe au ruisseau Provost. Ce point fut baptisé St-Malo selon les cartes de l'époque, cependant nous n'avons pas la preuve documentaire que ce soit Cartier qui lui ait donné ce nom, même si c'est vraisemblable.

8 - Où se trouvait située la ville d'Hochelaga que Cartier a visitée ? Si nous suivons le ruisseau Provost, ${ }^{65}$ nous constatons que celui-ci prend sa source dans la montagne dans la partie

61 H.P. Biggar, The Voyages of Jacques Cartier (Ottawa, 1924), 169. 62 Voir note 42.

63 Marcel Trudel, Atlas historique du Canada français (Les Presses de l'Université Laval, 1961), 21. 264-265.

64 H. P. Biggar, The Voyages of Jacques Cartier (Ottawa, 1924),

65 Voir carte du ruisseau Provost. 


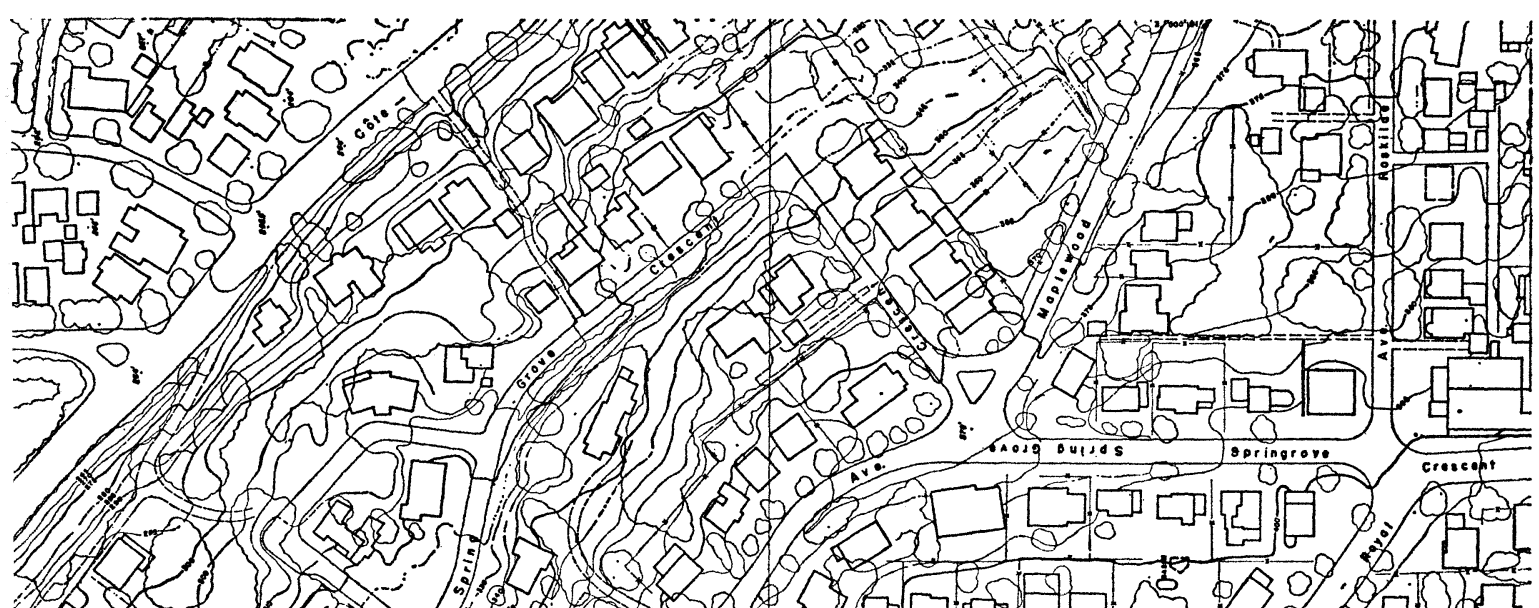
3. 3) P 40 . (a)

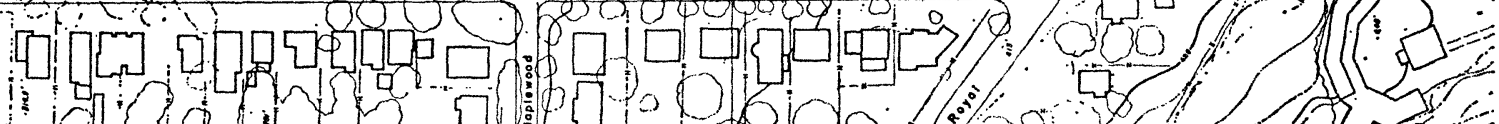

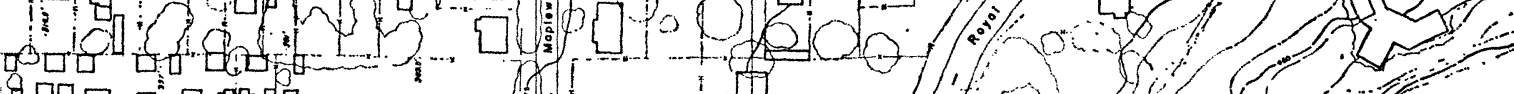

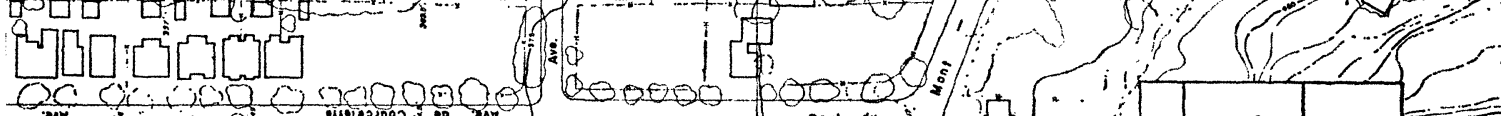

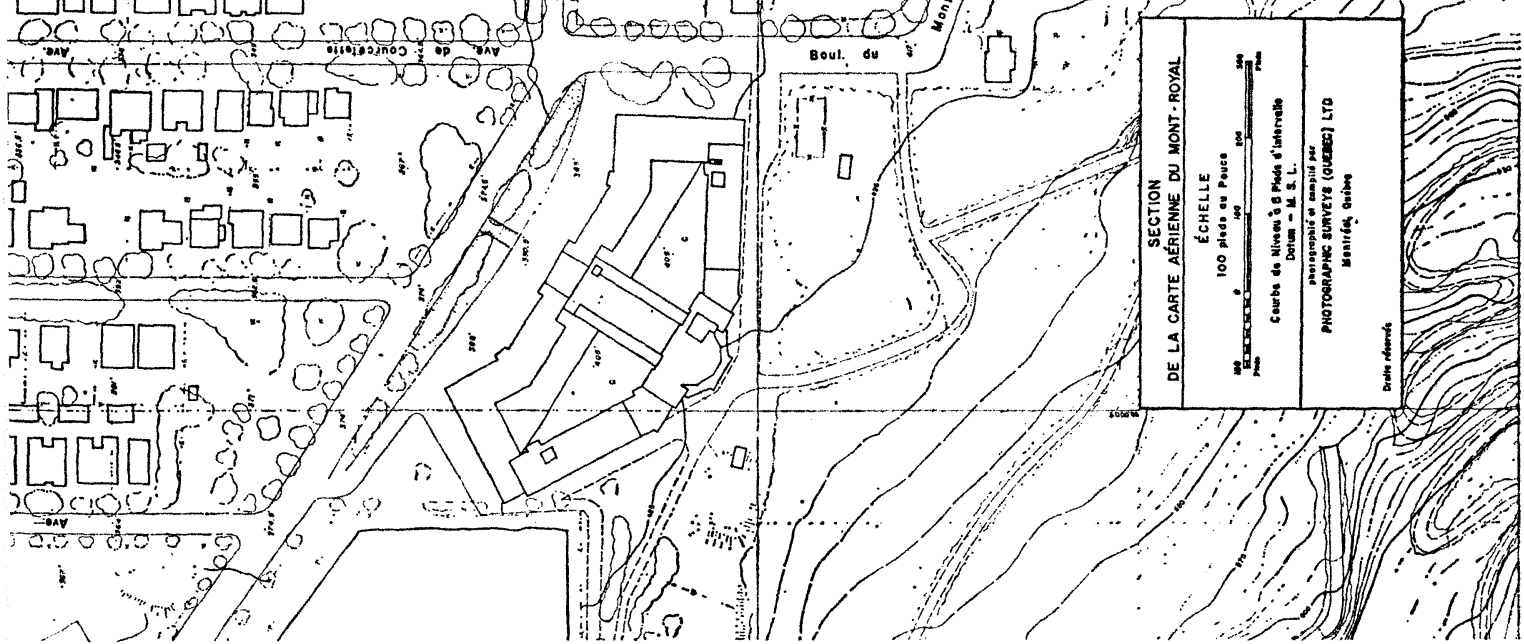


connue aujourd'hui sous le nom de cimetière Mont-Royal ou protestant. En 1535, Cartier, de son point de débarquement au ruisseau Provost, marcha une lieue et demie, avant d'être arrêté par un des chefs indiens qui lui tint un discours. Si on mesure en suivant les méandres peu nombreux de ce ruisseau, on constate que le point d'arrêt correspond à ce qui est aujourd'hui le parc situé entre les rues St-Laurent et Clark au sud de la rue St-Zotique, et cela grâce à la carte officielle des ruisseaux dressée par les ingénieurs et les arpenteurs de la ville de Montréal. ${ }^{66}$

De ce parc, si on parcourt encore une demi-lieue, comme l'indique Cartier, tout en suivant le ruisseau Provost, nous pourrons situer la ville d'Hochelaga d'une façon précise. En effet, selon la coutume des Iroquois, leur bourgade devait être située près d'un cours d'eau assez abondant et qui pouvait fournir l'eau potable toute l'année. De plus les Iroquois choisissaient toujours pour leur établissement un endroit facilement défendable et difficilement accessible aux assiégeants, tout en leur permettant d'observer, soit directement, soit par des sentinelles placées tout près du village, les allées et venues des étrangers.

En outre, il nous a fallu tenir compte des données de Cartier pour qui, Hochelaga lui a paru une ville toute ronde et contenant une cinquantaine de maisons, plus une place publique, ce qui donnerait approximativement 950 pieds de diamètre.

Quel était l'endroit le plus près possible du ruisseau, susceptible d'avoir un terrain assez plat et qui pouvait répondre à la manière de construire des Iroquois ? Nous avons consulté tout d'abord des photographies aériennes de la montagne, ${ }^{67}$ puis nous avons soumis le problème à un arpenteur-géomètre de la ville. Celui-ci, après avoir fait une étude des niveaux, c'est-à-dire des

66 Carte officielle des ruisseaux et fossés de Montréal, conservée au Service des Travaux publics de la Ville de Montréal, département des Eaux et Assainissements, sous la cote 268.

67 Cartes aériennes du Mont-Royal, conservées au Service des Travaux publics de la Ville de Montréal, Bureau du Registre des Rues (Plans et Archives), sous les numéros de cotes $227-30 ; 228-30$. A noter que la Ville de Montréal et la Cie "Photographic Surveys (Quebec) Ltd., en détiennent les droits d'auteur. 
élévations de terrain, en est venu à la conclusion que le seul endroit possible pour la construction d'une telle ville, était dans les limites suivantes: côté sud de la rue Maplewood entre l'avenue Courcelette et un peu à l'est de l'avenue Duchastel. En effet la pente du terrain à cet endroit, prise soit dans la direction nordsud ou est-ouest, ne varie pas de plus de 2 à $3 \%$ sur une distance de 950 pieds, soit le diamètre d'Hochelaga. ${ }^{68}$

9 - Cartier nous dit aussi que la ville n'avait qu'une entrée. Est-il possible de la localiser ? Nous croyons que les Indiens étaient des gens pratiques: aussi ils prévoyaient que pendant l'hiver leur porte d'entrée devait être libre de neige de telle sorte qu'ils ne soient pas prisonniers à l'intérieur de leur palissade. Pour cette raison nous présumons qu'elle était placée du côté du sud-est, c'est-à-dire du côté où il tombe le moins de neige ${ }^{69}$ et tout près du ruisseau.

10 - Cartier nous dit qu'après avoir quitté Hochelaga, il fit un quart de lieue dans la montagne et qu'il nomma celle-ci MontRoyal. Où situer ce point ? Nous dirons que c'est à l'ouest de l'école Polytechnique actuelle. En effet, après être allé sur place, vérifier à l'aide d'instrument ce que nous décrit Cartier, il ne nous fait aucun doute que ce point ne peut-être que dans cette direction, puisque de là, il nous est impossible de voir en même temps la rivière des Prairies et le fleuve St-Laurent. C'est ce qui explique que Cartier n'a pu découvrir l'insularité de Montréal.

11 - Quand en 1541, Cartier nous parle de la ville de Tutonaguy, nous croyons que c'est un autre nom pour désigner Hochelaga. En effet, parti de Charlesbourg-Royal le 7 septembre 1541, Cartier nous dit qu'il arriva le 11 septembre au premier

68 Illustration de la Bourgade d'Hochelaga en 1535, par Ernest Lemay. Cette illustration est la propriété de Claude Perrault. M. E. Lemay a exécuté ce plan d'après les données du manuscrit de Cartier et suivant les cartes aériennes et celle du ruisseau Provost. Sur cet item, voir, W.D. Lighthall, "The false plan of Hochelaga", Mémoires de la Société royale du Canada (1932), section II: 181-192. H.P. Biggar, The Voyages of Jacques Cartier (Ottawa, 1924), 144-145, a reproduit ce faux plan.

69 Rapport de l'Archiviste de la Province de Québec pour les années 1923-1924, 42. 
"sault", lequel est à deux lieues de distance de la ville de Tutonaguy. Donc, avant même de débarquer, Cartier situe cette bourgade à la même distance que celle d'Hochelaga. D'où nous pouvons déduire qu'il a appris avant d'arriver à Montréal ce changement de nom. Mais il n'en donne pas la raison, à moins que la partie perdue du manuscrit de 1541 n'en contienne la réponse.

12 - Quant aux données de Jacques Noël, il faut les traiter avec prudence, puisqu'il nous dit en 1587 que Montréal est située au $44^{\circ}$ de latitude ${ }^{70}$ tandis qu'en réalité c'est à $45^{\circ} 31^{\prime}$ de latitude nord, soit une différence de $1^{\circ} 31^{\prime}$ ou environ cent milles plus au sud. Sur l'identité de ce personnage, nous croyons que M. Marcel Trudel en a fait une bonne généalogie. ${ }^{71}$ Enfin quelle est l'origine du nom de Montréal ? Plusieurs auteurs ont soutenu différentes hypothèses. Quant à nous, nous croyons que la plus simple et partant la plus naturelle nous est donnée dans les relations des Jésuites de 1642. Nous pouvons y lire ceci: "Mais pour remonter à nostre Isle, je diray en passant que l'aspect d'vne belle montagne qui s'y rencontre, luy a fait porter le nom de Montreal au Mont-royal." 72 Un peu plus loin nous pouvons encore lire: "Après la feste (15 août 1642) on fut visiter les grands bois qui couurent cette Isle: et estans amenez à la montagne dont elle tire son nom"...73 Quant aux Indiens, les relations de 1642 nous disent ceci: "Les Sauvages, qui la nomment Minitik8ten entag8giban, l'Isle où il y auoit vne ville ou vne bourgade." 74

Quel est le jugement que nous pouvons porter sur l'œuvre de Cartier si ce n'est celui du père de la Nouvelle-France qui

70 H.P. Biggar, The Voyages of Jacques Cartier (Ottawa, 1924), 260. Voir aussi Don W. Thomson, L'Homme et les Méridiens (Ottawa, $1965)$, vol. I.

${ }^{71}$ Marcel Trudel, Histoire de la Nouvelle-France - I - Les Vaines tentatives 1524-1603 (Montréal, 1963), 222. II $: 36$.

72 Relations des Jésuites (3 vol., éd. C.-H. Laverdière, Québec, 1858),

73 Ibid., 38.

74 Ibid., 36. 
dit: "Quartier en eust aussi bien fait le discours (de ses découvertes) pour le laisser à la posterité comme il l'a fait de tout ce qu'il a veu \& descouuert." 75

En terminant permettez-nous de formuler le vœu qu'un jour le manuscrit de 1541 soit retrouvé. Nous espérons que cette étude de la découverte de Montréal contribuera à faire mieux connaître les débuts d'Hochelaga.

Claude Perrault

${ }^{75}$ Oeuvres de Champlain (éd. abbé C.-H. Laverdière, Québec, 1870, 5 vol.), V: 158 . 